\title{
Significant pain relief with loading dose zoledronic acid in bone metastases is only seen in patients with elevated initial serum $C$ telopeptide (CTX)
}

This article was published in the following Dove Press journal:

Pragmatic and Observational Research

27 July 201 I

Number of times this article has been viewed

\section{J Dekoninck' \\ F Geurs' \\ R De Loecker ${ }^{2}$ \\ Y Deprest ${ }^{2}$}

'Department of Medical Oncology, ${ }^{2}$ Laboratory, Regional Hospital Sint Maria, Halle, Belgium
Correspondence: F Geurs Department of Medical Oncology, Regional Hospital Sint Maria, Ziekenhuislaan 100, 1500 Halle, Belgium Tel +32 23636610

Email g@regzhsintmaria.be

\begin{abstract}
In symptomatic bone metastases with significant pain, refractory to standard analgesics and radiotherapy, loading dose zoledronic acid (ZA) represents a simple and nontoxic treatment to obtain significant pain relief in a very short time. Its analgesic effect is limited to patients with massive osteoclast activation with high initial serum C-telopeptide (CTX). The pain reduction is proportionally correlated with the reduction of CTX.
\end{abstract}

Keywords: bone resorption, bisphosphonates, CTX levels, VAS score

\section{Introduction}

Bone is a frequent site of metastasis of several types of solid tumors, and metastatic bone disease is the most frequent cause of pain in cancer patients. ${ }^{1}$ Solid tumors that most frequently produce bone metastases are breast, prostate $(75 \%-85 \%)$ and lung $(40 \%)$ tumors. ${ }^{2}$ The presence of bone metastases significantly changes the course of the diseases and, if left untreated, may produce skeletal-related events (SRE) that greatly reduce quality of life and may even lead to death. Median survival rates from the time that metastatic bone disease is diagnosed range from 12 to 53 months, depending on the type of primary tumor. ${ }^{3}$

Bone metastases occur as a consequence of excessive osteoclastic activity rather than from direct tumor-cell infiltration. Tumor cells release factors that stimulate osteoclastic and osteoblastic activity, disrupting the balance between osteoclastmediated bone resorption and osteoblast-mediated bone formation and repair. ${ }^{4}$ As a result of accelerated bone turnover, bone resorption predominates, leading to loss of the bone's structural integrity and development of SRE. One of these changes includes breakdown of type I collagen transverse cross-links, the main constituent of the bone's organic matrix, resulting in the release of degradation molecules such as pyridinoline, deoxypyridinoline, N-telopeptides (NTX), and C-telopeptides (CTX). ${ }^{5}$ These products are released into the blood and excreted in the urine, and constitute markers of bone resorption and formation (for example calcium, hydroxypyridinoline, NTX, CTX, pyridinoline, deoxypyridinoline, and bone sialoprotein) or bone formation alone (for example alkaline phosphatase and osteocalcin). The detection of these markers in blood or urine is associated with the presence and progression of bone metastases and is linked to prognosis and response to pharmacological treatment. ${ }^{6}$ Using a histomorphometric analysis, Delmas et $\mathrm{al}^{7}$ demonstrated that urinary excretion of several degradation products of type I collagen is closely related to bone resorption rate. 
The treatment of bone metastases is multidisciplinary, and bisphosphonates play a major role as they are inhibitors of osteoclastogenesis and osteoclast-mediated bone resorption. For this reason, they have been used since the 1960s for treating diseases associated with increased bone resorption activity, such as Paget's disease and osteoporosis. Their chemical structure is similar to that of inorganic pyrophosphates, the bone's mineralization regulators. ${ }^{8}$

Zoledronic acid (ZA) is a third-generation bisphosphonate with an antiresorptive potency about a thousand times greater than second-generation bisphosphonates. ${ }^{9}$ ZA accumulates in bone matrix and is released during bone resorption. Its mechanism of action involves inhibition of osteoclast maturation and migration, induction of osteoclast apoptosis through inhibition of protein prenylation via the mevalonate pathway ${ }^{10}$ and reduction of interleukin 6 (IL-6) secretion, and it seems to have direct antitumor and anti-angiogenic properties. ${ }^{11} \mathrm{ZA}$ is the most widely used bisphosphonate in the treatment of bone metastases, and its use in this condition is supported by numerous clinical trials demonstrating its superiority over pamidronate and placebo. Of these, the most significant findings are those obtained in two Phase III studies carried out by Rosen et al in multiple myeloma and breast cancer, ${ }^{12}$ lung cancer and other solid tumors, ${ }^{13}$ and a Phase III study by Saad et al in prostate cancer. ${ }^{14}$ In these trials, ZA was demonstrated to significantly reduce skeletal morbidity, the number of SRE, the proportion of patients developing SRE, and the delay in time to first SRE. Moreover pain control was improved. In addition, results of these trials suggest that assessment of bone resorption markers, such as CTX, can be an effective approach in identifying patients at high risk of developing bone metastases and in preventing SRE of which pain is the most frequently presenting symptom. CTX has proved to be a useful marker to predict disease progression, expressed by SRE, ${ }^{15}$ but there is no study directly correlating the CTX levels with pain in patients with proven painful bone metastases.

Taking into account all these concerns, we carried out a prospective, nonrandomized study to evaluate whether serum CTX levels may be useful markers to predict the analgesic effect of ZA in patients with solid tumors and confirmed bone metastases.

\section{Patients and methods Study design}

This was a prospective, nonrandomized trial performed at the Oncology Department of the AZ Sint Maria Hospital in Halle, Belgium. Patients were recruited between August 2010 and April 2011. The study protocol was approved by the hospital's Ethics Committee, and all procedures were conducted in accordance with Good Clinical Practice Guidelines and with the Declaration of Helsinki of 1975, as revised in 2000. All patients provided their written informed consent.

\section{Study procedures}

Consecutive patients with confirmed painful bone metastases secondary to a solid tumor which was diagnosed at the Oncology Department of our hospital, were selected to participate and asked for informed consent. No other exclusion criteria were used. After informed consent, the visual analog score (VAS) was taken by a palliative nurse and blood samples were taken for detection of serum CTX, serum creatinine, and serum urea. All patients were then treated with $4 \mathrm{mg}$ of intravenous ZA for 3 days consecutively. A new comparative blood sample was taken the day after the 3 days of ZA administration.

CTX dosage was done using Elecsys ${ }^{\circledR} \beta$-CrosslapsTM/ Serum assay (Roche Diagnostics, Basel, Switzerland). Precision was evaluated on a two-level quality control (Level 1: at $285 \mathrm{pg} / \mathrm{mL}$ with a c.v. of 2,77. Level 2: at $638 \mathrm{pg} / \mathrm{mL}$ with a c.v. of 2.45).

Adverse events (AE) and severe AE (SAE) were recorded throughout the whole study period.

\section{Statistical analysis}

Statistical analyses were carried out using SPSS (v 12.0; SPSS Inc, Chicago, IL). Qualitative variables were expressed as percentages, and continuous variables were described using the mean and the standard deviation (SD).

Frequency distributions and relationships between variables were compared using Student's $t$-test, MannWhitney U test, one-sided analysis of variance (ANOVA) and Wilcoxon signed-rank test.

\section{Results}

\section{Patient characteristics}

Between August 2010 and February 2011, 20 patients with solid tumors and confirmed bone metastases were included in the study. Baseline patient characteristics are shown in Table 1. Mean age was 74 (range 44-90) years. There were ten male and ten female patients and the primary tumor was located in the: prostate (25\%); breast (15\%); lung (15\%); bladder or kidney (10\%); gastrointestinal tract (15\%); and hematological neoplasms (20\%). At diagnosis, 16 (80\%) 
Table I Baseline patient characteristics $(n=20)$

\begin{tabular}{lll}
\hline Characteristics & No. & Percent \\
\hline Age, years & $74 \pm 12(44-90)$ & \\
$\quad$ Mean \pm SD (range) & 10 & 50 \\
Gender & 10 & 50 \\
$\quad$ Male & & \\
$\quad$ Female & 5 & 25 \\
Tumor localization & 3 & 15 \\
$\quad$ Prostate & 2 & 10 \\
Breast & 3 & 15 \\
Bladder/kidney & 4 & 20 \\
Lung & 3 & 15 \\
$\quad$ Myeloid/lymphoid & \\
$\quad$ Gastrointestinal &
\end{tabular}

Abbreviation: SD, standard deviation.

patients had five or more bone metastases, and eight (40\%) had received palliative radiotherapy to relieve bone pain.

\section{CTX levels}

At the baseline, mean serum CTX levels were $896.3 \pm$ $658.7 \mathrm{pg} / \mathrm{dL}$ (Table 2). CTX levels significantly decreased after the 3-day scheme of ZA daily $(227.5 \pm 162.7 \mathrm{pg} / \mathrm{dL}$; $P<0.001$, Table 3).

At the baseline, there were no significant differences between CTX levels observed in male and female patients $(1088.3 \pm 262.8$ vs $704.2 \pm 119.8 \mathrm{pg} / \mathrm{dL} ; P=0.41)$. Neither age nor type of tumor have any influence on the level of CTX ( $P=0.236$ and $P=0.343$, respectively). Gender, age, and type of tumor had no impact on the degree of CTX reduction due to the treatment (Table 2).

\section{VAS score}

At the baseline, mean VAS score was $7.95 \pm 1.67$ with a semirepresentative right-sided Gaussian distribution. (Figure 1). VAS score significantly decreased after the 3-day scheme of ZA daily (3.39 $\pm 2.75 ; P<0.001$, Table 3$)$, illustrated by the alteration to a non-Gaussian distribution due to the predominance of lower VAS scores (Figure 1).

At the baseline, there was no significant difference between VAS score levels observed in male and female

Table $2 P$-values - baseline and reduction of CTX by age, gender or type of tumor

\begin{tabular}{llll}
\hline & Age & Gender & Type of tumor \\
\hline Baseline level CTX & $P=0.236$ & $P=0.4 I$ & $P=0.343$ \\
Absolute reduction CTX & $P=0.236$ & $P=0.392$ & $P=0.339$ \\
Percentage reduction CTX & $P=0.24 I$ & $P=0.329$ & $P=0.523$
\end{tabular}

Abbreviation: CTX, C-telopeptides.
Table 3 Changes in serum markers and VAS score during ZA treatment

\begin{tabular}{lccl}
\hline Measurements & $\begin{array}{l}\text { Baseline } \\
\text { mean (SD) }\end{array}$ & $\begin{array}{l}\text { After ZA } \\
\text { mean (SD) }\end{array}$ & P value* \\
\hline Serum markers & & & \\
CTX (pg/dL) & $896.3 \pm 658.7$ & $227.5 \pm 162.7$ & $<0.00 \mathrm{I}$ \\
Creatinine (mg/dL) & $0.95 \pm 0.34$ & $0.83 \pm 0.27$ & 0.06 \\
Urea (mg/dL) & $46.0 \pm 13.5$ & $34.2 \pm 9.3$ & 0.005 \\
VAS & $7.95 \pm 1.67$ & $3.39 \pm 2.75$ & $<0.00 \mathrm{I}$ \\
\hline
\end{tabular}

Note: *Statistically significant $(P<0.05)$ using paired sample $t$-test.

Abbreviations: CTX, C-telopeptides; ZA, zoledronic acid; SD, standard deviation; VAS, visual analog score.

patients $(7.7 \pm 0.6$ vs $8.2 \pm 0.5 ; P=0.605)$. Neither age nor type of tumor had any influence on the baseline VAS score ( $P=0.332$ and $P=0.238$, respectively; Table 4$)$. Gender, age, and type of tumor had no impact on the degree of CTX reduction due to the treatment (Table 4).

\section{Correlations between CTX and VAS}

Decrease in VAS and CTX was measured by two methods: (1) measurement of the absolute reduction, ie, the difference in level before and after ZA treatment; (2) reduction in terms of percentage calculated from the proportion of the levels of VAS and CTX before and after ZA treatment.

There was a significant correlation between the decrease measured in VAS score levels and the decrease in CTX level measured via absolute reduction $(P=0.021)$ and percentage reduction $(P=0.002)$.

There was a trend to a significant correlation between the percentage reduction of VAS score and the baseline CTX level $(P=0.084)$.

\section{Safety}

No decrease in renal function was recorded after ZA treatment. On the contrary, we observed a slight increase in renal function with almost significant values (Table 3).

No severe adverse effects were recorded during the period of treatment. All patients were advised to consult a dentist to prevent osteonecrosis of the jaw.

\section{Discussion}

The patient group was small but representative for a general oncology population.

All patients had a high VAS score before ZA treatment. VAS score dropped regardless of the primary type of tumor. VAS score is still a subjective marker for pain, but is internationally accepted for pain evaluation. 

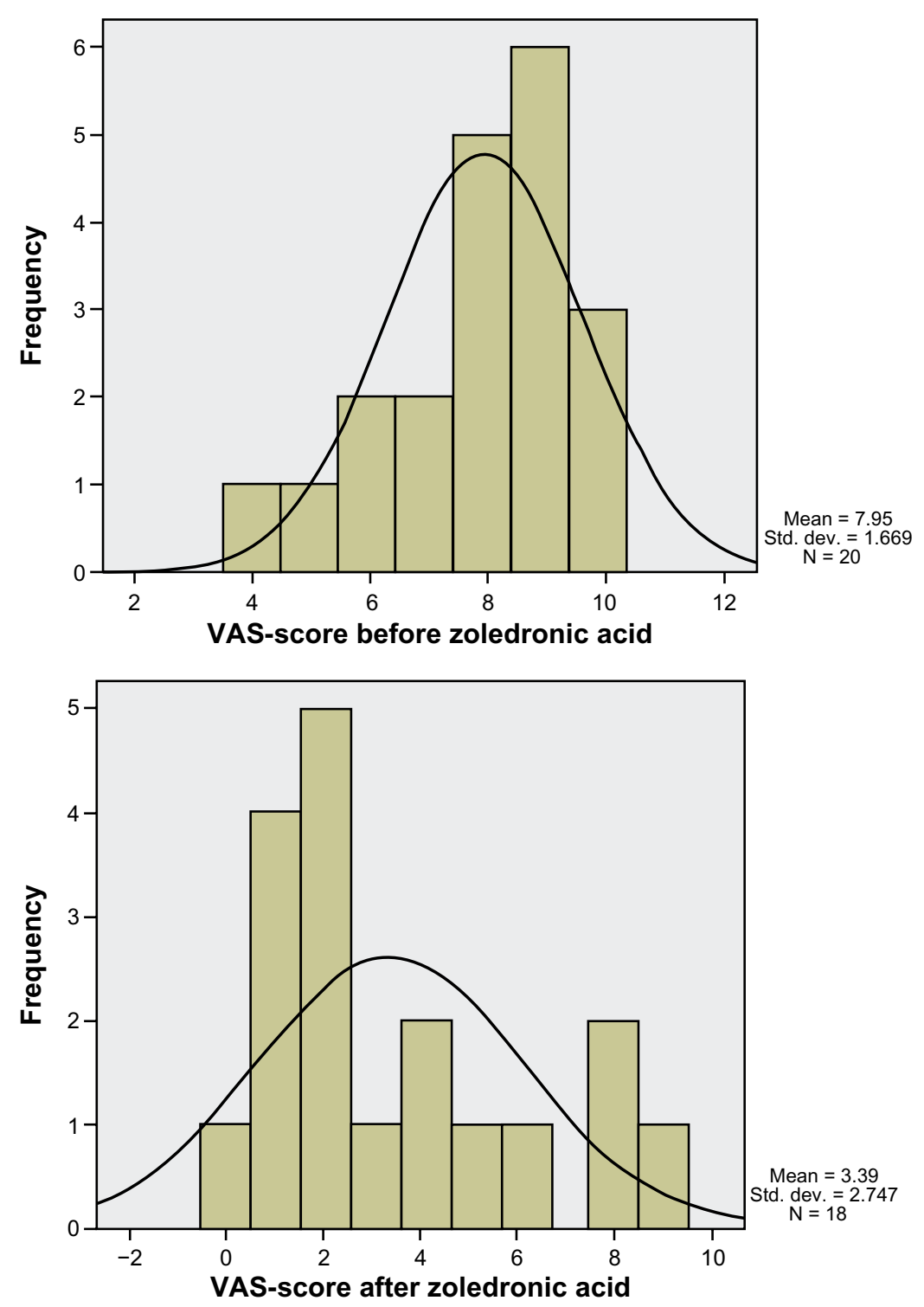

Figure I Distribution of VAS score before and after ZA treatment.

Abbreviations: ZA, zoledronic acid; VAS, visual analog score.

Analgesic consumption was unchanged during treatment. If analgesic treatment was insufficient after the 4-day trial, the dosage of analgesics was increased.

Bisphosphonates are known to be nephrotoxic. There was no decrease in renal function measured during the administration of ZA. All patients were hospitalized and received an adequate hydration scheme adapted to their cardiac, renal, and hydration status. This may be the explanation for the slight increase in renal function during treatment. We therefore conclude that this treatment schedule of ZA with an adapted hydration scheme has no effect on renal function.

The strong correlation $(P=0.002)$ between the percentage reduction of VAS and the percentage reduction of CTX proves that the initially elevated and subsequent reduction of CTX can be used as an objective predictive marker for pain reduction due to bisphosphonates. Such an objective biochemical correlation between CTX decrease and pain relief in patients with bone metastases is a new finding in oncology.

There is a tendency for a significant correlation between high baseline CTX and subsequent pain relief due to bisphosphonates $(P=0.084)$. This implies that even isolated elevated baseline CTX levels are possibly useful as a predictor of pain relief. We are convinced that a larger study population would probably demonstrate the statistical significance. 
Table $4 P$-values - baseline and reduction of VAS by age, gender or type of tumor

\begin{tabular}{llll}
\hline & Age & Gender & Type of tumor \\
\hline Baseline VAS & $P=0.332$ & $P=0.605$ & $P=0.238$ \\
Absolute reduction VAS & $P=0.294$ & $P=0.419$ & $P=0.215$ \\
Percentage reduction VAS & $P=0.262$ & $P=0.528$ & $P=0.246$ \\
\hline
\end{tabular}

Abbreviation: VAS, visual analog score.

Table 5 CTX and VAS during treatment - data from three patients

\begin{tabular}{llllll}
\hline & \multicolumn{2}{l}{ CTX $(\mathbf{p g} / \mathbf{d L})$} & & \multicolumn{2}{l}{ VAS (0-10) } \\
\cline { 2 - 3 } & Baseline & After ZA & & Baseline & After ZA \\
\hline Patient I & 208 & 211 & & 9 & 8 \\
Patient 2 & 369 & 360 & & 9 & 9 \\
Patient 3 & 792 & 233 & & 8 & 8
\end{tabular}

Abbreviations: CTX, C-telopeptides; ZA, zoledronic acid; VAS, visual analog score.

Its clinical relevance is further corroborated by the analysis of the two of our patients with low CTX values without analgesic effect from bisphosphonates (patient 1 and 2; Table 5).

Another patient with a baseline CTX of $792 \mathrm{pg} / \mathrm{dL}$ demonstrated no analgesic effect (patient 3; Table 5). This may be explained by the presence of brain metastases in this patient, which probably caused central and neuropathic pain.

\section{Conclusion}

The analgesic effect of bisphosphonates on pain caused by bone metastases is well documented. The dose and frequency of bisphosphonate treatment is subject to much research. For the first time, the administration of a loading dose of ibandronate demonstrated significant pain relief. ${ }^{16}$

Following the same treatment plan, we applied a 3-day schedule of administration of ZA which resulted in a similar reduction of pain scores.

The originality of our work comes from the initial documentation of important bone destruction as illustrated by the high CTX prior to ZA administration. The subsequent significant decrease of CTX after ZA administration paralleled the decrease in VAS score.

The important role of CTX decrease as a necessary marker for ZA-mediated pain relief was further corroborated by the fact that in two of the patients with neoplasia, but without elevated CTX, the administration of a loading dose of ZA did not give any significant pain relief.
Other authors have also seen significant pain relief and decrease of VAS score by a weekly administration of a lower dose of ZA. ${ }^{17}$

Our study was the first to show a rapid decrease of CTX after 3 days' infusion of the daily dose of ZA.

In summary, the loading dose principle ( 3 consecutive days of $4 \mathrm{mg}$ ZA administration) permits rapid pain relief in patients with bone metastases. This rapid pain relief is especially seen in those patients with elevated CTX before starting zoledronic treatment. The subsequent pain relief paralleled the decrease in CTX, illustrating the importance of osteoclast inhibition.

\section{Disclosure}

The authors report no conflicts of interest in this work.

\section{References}

1. Coleman RE. Clinical features of metastatic bone disease and riskof skeletal morbidity. Clin Cancer Res. 2006;12(20 Pt 2): 6243-6249.

2. Mundy GR. Metastasis to bone: causes, consequences and therapeutic opportunities. Nat Rev Cancer. 2002;2(8):584-593.

3. Coleman RE. Metastatic bone disease: clinical features, pathophysiology and treatment strategies. Cancer Treat Rev. 2001;27(3): $165-176$.

4. Mundy GR, Guise TA. Pathophysiology of bone metastasis. In: Rubens RD, Mundy GR, editors. Cancer and the skeleton, 3rd ed. London, UK: Informa Healthcare;2000:43-44.

5. Ulrich U, Rhiem K, Schmolling J, et al. Cross-linked type I collagen $\mathrm{C}$ - and N- telopeptides in women with bone metastases from breast cancer. Arch Gynecol Obstet. 2001;264(4):186-190.

6. Lipton A, Costa L, Ali S, Demers L. Use of markers of bone turnover for monitoring bone metastases and the response to therapy. Semin Oncol. 2001;28(4 Suppl 11):54-59.

7. Delmas PD, Schlemmer A, Gineyts E, Riis B, Christiansen C. Urinary excretion of pyridinoline crosslinks correlates with bone turnover measured on iliac crest biopsy in patients with vertebral osteoporosis. J Bone Miner Res. 1991;6(6):639-644.

8. Kavanagh KL, Guo K, Dunford JE, et al. The molecular mechanism of nitrogen-containing bisphosphonates as antiosteoporosis drugs. Proc Natl Acad Sci U S A. 2006;103(20):7829-7834.

9. Wilder L, Jaeggi KA, Glatt M, et al. Highly potent geminal bisphosphonates. From pamidronate disodium (Aredia) to zoledronic acid (Zometa). J Med Chem. 2002;45(17):3721-3738.

10. Yu J, Chang SS, Suratwala S, et al. Zoledronate induces apoptosis in cells from fibro-cellular membrane of unicameral bone cyst (UBC). J Orthop Res. 2005;23(5):1004-1012.

11. Green JR. Antitumor effects of bisphosphonates. Cancer. 2003; 97(Suppl 3):840-847.

12. Rosen LS, Gordon D, Kaminski M, et al. Zoledronic acid versus pamidronate in the treatment of skeletal metastases in patients with breast cancer or osteolytic lesions of multiple myeloma: a phase III, double-blind, comparative trial. Cancer J. 2001;7(5): 377-387.

13. Rosen LS, Gordon D, Tchekmedyian S, et al. Zoledronic acid versus placebo in the treatment of skeletal metastases in patients with lung cancer and other solid tumors: a phase III, double-blind, randomized trial - The Zolendronic Acid Lung Cancer and Other Solid Tumors Study Group. J Clin Oncol. 2003;21(16):3150-3157. 
14. Saad F, Gleason DM, Murray R, et al. A randomized, placebo-controlled trial of zoledronic acid in patients with hormone-refractory metastatic prostate carcinoma. J Natl Cancer Inst. 2002;94(19):1458-1468.

15. Lopez-Carrizosa MC, Samper-Ots PM, Pérez AR. Serum C-telopeptide levels predicts the incidence of skeletal related events in cancer patients with secondary bone metastases. Clin Transl Oncol. 2010; 12(8):568-573.
16. Mancini I, Dumon JC, Body JJ. Efficacy and safety of ibandronate in the treatment of opioid-resistant bone pain associated with metastatic bone disease: a pilot study. J Clin Oncol. 2004;22(17):3587-3592.

17. Xinmin Z, Xiaofeng X, Lin G. Biomarker alterations with metronomic use of low-dose zoledronic acid for breast cancer patients with bone metastases and potential clinical significance. Breast Cancer Res Treat. 2010;124:733-743.

Pragmatic and Observational Research

Dovepress

\section{Publish your work in this journal}

Pragmatic and Observational Research is an international, peer-reviewed, open access journal that publishes data from studies designed to reflect more closely medical interventions in real-world clinical practice compared with classical randomized controlled trials (RCTs). The manuscript management system is completely online and includes a very quick and fair peer-review

Submit your manuscript here: http://www.dovepress.com/pragmatic-and-observational-research-journal

system. Visit http://www.dovepress.com/testimonials.php to read real quotes from published authors. 